\title{
Zn(Mn)O Surface Alloy Studied by Synchrotron Radiation Photoemission
}

\author{
E. Guziewicz ${ }^{a}$, K. Kopalko ${ }^{a}$, J. SAdowski ${ }^{a}$, M. GuzIEwicz ${ }^{b}$ \\ AND Z. GOLACKI ${ }^{a}$ \\ ${ }^{a}$ Institute of Physics, Polish Academy of Sciences \\ al. Lotników 36/42, 02-688 Warszawa, Poland \\ ${ }^{b}$ Institute of Electron Technology \\ al. Lotników 36/42, 02-688 Warszawa, Poland
}

\begin{abstract}
The $\mathrm{Mn} / \mathrm{ZnO}(000 \underline{1})$ system was investigated by synchrotron radiation photoemission. The $\mathrm{Mn} / \mathrm{ZnO}$ interface with $4 \mathrm{ML}$ of manganese deposited onto the $\mathrm{ZnO}$ surface was annealed up to $500^{\circ} \mathrm{C}$. No Mn capping layer was found at the surface after annealing as was confirmed by scanning Auger spectroscopy experiment. We used a resonant photoemission to extract the Mn3d partial density of states in photoemission spectra. The Mn3d states contribute to the electronic structure of the system within $10 \mathrm{eV}$ of the Fermi level. They show three features: a main peak at $3.8-4.5 \mathrm{eV}$, a valence structure at the top of the valence band $(1-3 \mathrm{eV})$, and a broad satellite situated between 5.5 and $9 \mathrm{eV}$ below $E_{\mathrm{F}}$. The satellite/main branching ratio was determined to be 0.43 , which is a fingerprint of strong hybridization between the Mn3d electrons and the valence band of the crystal. The hybridization effect in $\mathrm{Zn}_{1-x} \mathrm{Mn}_{x} \mathrm{O}$ surface alloy is comparable to $\mathrm{Zn}_{1-x} \mathrm{Mn}_{x} \mathrm{~S}$ and much higher than in $\mathrm{Zn}_{1-x} \mathrm{Mn}_{x} \mathrm{Se}, \mathrm{Zn}_{1-x} \mathrm{Mn}_{x} \mathrm{Te}$, and $\mathrm{Ga}_{1-x} \mathrm{Mn}_{x}$ As semimagnetic compounds.
\end{abstract}

PACS numbers: 73.20.--r, 79.60.--i, 79.60.Jv

\section{Introduction}

The wide band gap semiconductor $\mathrm{ZnO}$ containing ions of the $3 d$ transition metals $(\mathrm{TM})$ is a promising material for short wavelength magneto-optical applications. Manganese ions are usually incorporated in situ during $\mathrm{ZnO}$ by different methods like flux growth [1], combinatorial laser molecular-beam epitaxy method [2] or pulsed-laser deposition [3]. 
In the experiment presented here we used the method of Mn diffusion into the $\mathrm{ZnO}$ single crystal matrix after annealing up to $500^{\circ} \mathrm{C}$ of the $\mathrm{Mn} / \mathrm{ZnO}$ interface with $4 \mathrm{ML}$ of manganese deposited onto the $\mathrm{ZnO}(000 \underline{1})$ surface. We used resonant photoemission method to investigate the Mn3d contribution to the valence band in order to determine interactions between the magnetic component and the valence band of the $\mathrm{ZnO}$ single crystal. The resonant-photoemission spectra were taken across the Mn3p-Mn3d absorption threshold (photon energy between 46 and $60 \mathrm{eV})$. At a resonance the $\mathrm{Mn} 3 p$ electron is excited into the empty part of the Mn3d states. This excited state decay via autoionization in which the Mn3d electron drops down to fill the Mn3 $p$ hole and its energy is transferred to another Mn3d electron that is ejected into the continuum:

$$
\mathrm{Mn} 3 p^{6} 3 d^{5}+h \nu \rightarrow \operatorname{Mn} 3 p^{5} 3 d^{6} \rightarrow \operatorname{Mn} 3 p^{6} 3 d^{4}+\mathrm{e} .
$$

The final state of the process is the same like in direct photoemission from the Mn3d shell:

$$
\mathrm{Mn} 3 p^{6} 3 d^{5}+h \nu \rightarrow \operatorname{Mn} 3 p^{6} 3 d^{4}+\mathrm{e} .
$$

The interference between the two channels of photoemission leads to an increase in the photoemission intensity as the photon energy is swept through the $3 p-3 d$ threshold. The asymmetric Fano line shape characterizes the intensity variations. We measured photoemission spectra (energy distribution curves - EDCs) at a maximum and a minimum of the Fano resonance, which for manganese are $51 \mathrm{eV}$ and $47 \mathrm{eV}$, respectively. Calculated difference spectra $\triangle \mathrm{EDC}(\mathrm{EDC}(51 \mathrm{eV})$ minus $\mathrm{EDC}(47 \mathrm{eV}))$ is related to the Mn3d partial density of states.

\section{Experimental conditions}

The single $\mathrm{ZnO}$ wurzite crystal was grown in the Institute of Physics, Polish Academy of Sciences by chemical vapor transport method using chlorine as transporting agent. The orientation of the crystal was determined by X-ray diffraction. A clean (0001) surface of $\mathrm{ZnO}$ monocrystal was prepared in an ultra-high vacuum system $\left(p \approx 4 \times 10^{-10}\right.$ Torr $)$ using argon ion bombardment $(U=600 \mathrm{~V}$, $t=40 \mathrm{~min})$ and annealing to $300^{\circ} \mathrm{C}$. The experiment was performed at BL41 beamline at the MAX I storage ring in Lund, Sweden. Angle-resolved photoemission spectra were recorded with the overall energy resolution of $200 \mathrm{meV}$ at photon energy of $50 \mathrm{eV}$. Manganese was deposited in the III-Mn-V MBE system vacuum connected to the photoemission chamber. During deposition all the sources other than Mn were cooled down to low temperatures. The Mn coverage of the $\mathrm{ZnO}$ surface was estimated from precise calibrations of $\mathrm{Mn}$ flux used for III-Mn-V MBE growth [4] We sequentially deposited small amounts (from 0.2 to $4 \mathrm{ML}$ ) of manganese on a clean $\mathrm{ZnO}(000 \underline{1})$ surface under ultra-high vacuum and, via photoemission spectra, we follow changes in the valence band of 
$\mathrm{Mn} / \mathrm{ZnO}$ system and in the experimental Mn3d partial density of states. After deposition of $4 \mathrm{ML}$ of manganese the $\mathrm{Mn} / \mathrm{ZnO}$ system was heated up to $500^{\circ} \mathrm{C}$ in order to promote diffusion of manganese atoms. The photoemission spectra were taken at room temperature, normalized to the photon flux and the secondary electron background has been subtracted. The binding energy scale is referred to the Fermi level of a thick manganese film. All measurements were performed at room temperature.

\section{Experimental results}

A set of EDCs were taken after each step of deposition at various photon energies between 30 and $110 \mathrm{eV}$. In Fig. 1 we present normal emission EDCs taken for photon energy of $51 \mathrm{eV}$ (maximum of Fano resonance for manganese) within $14 \mathrm{eV}$ of the Fermi level $\left(E_{\mathrm{F}}\right)$. This binding energy range covers the valence band (0-9 eV below $\left.E_{\mathrm{F}}\right)$ and the Zn3d core level emission (9-12 eV). All the spectra in

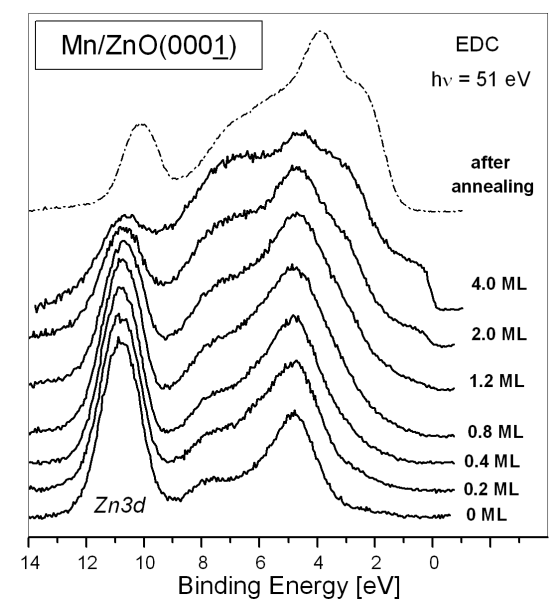

Fig. 1. EDCs for $\mathrm{Mn} / \mathrm{ZnO}(000 \underline{1})$ measured after each step of deposition and after annealing for $h \nu=51 \mathrm{eV}$ (a maximum of $\mathrm{Mn} 3 p-3 d$ resonance).

Fig. 1 were normalized to maximum to emphasize line-shape changes. The bottom spectrum shows clean $\mathrm{ZnO}(0001)$ valence band and $\mathrm{Zn} 3 d$ emission taken before $\mathrm{Mn}$ deposition. The spectrum was shifted by $0.12 \mathrm{eV}$ towards higher binding energies in order to compensate the binding energy shift due to a Shottky barrier formation. Contribution from the $\mathrm{ZnO}$ substrate is visible at $4.8 \mathrm{eV}$ up to the $4 \mathrm{Mn} \mathrm{ML}$ depositions. For $\Theta=4 \mathrm{ML}$ the position of this highest valence band peak is shifted about $0.3 \mathrm{eV}$ towards lower binding energy and results from contribution of the manganese emission in this binding energy region. The manganese contribution is also observed between 6.5 and $8 \mathrm{eV}$ starting from coverage of $1.2 \mathrm{ML}$. For $\Theta=2$ ML a metallic shoulder at the Fermi level is created. This means that parts 
of manganese atoms do not diffuse into the interface region and remain at the crystal surface. For $\Theta=4 \mathrm{ML}$ the structure between $6.5-8 \mathrm{eV}$ is more pronounced and a new shoulder is created at around $3.2 \mathrm{eV}$. The upper curve in Fig. 1 (dashed line) was taken after 18 hours of annealing in $500^{\circ} \mathrm{C}$ in the MBE chamber. After annealing the metallic shoulder at the Fermi energy has disappeared and the rest of photoemission spectra shifts towards lower binding energy. Binding energy of the $\mathrm{Zn} 3 d$ core level after annealing equals $10.1 \mathrm{eV}$, which is $0.6 \mathrm{eV}$ lower than for a clean $\mathrm{ZnO}$ single crystal. The main structure of the valence band is now at $3.8 \mathrm{eV}$ below the Fermi level and the spectra between $9 \mathrm{eV}$ and the Fermi edge is very similar to the Mn3d contribution in another II-VI based ternary compounds reported in the papers published before [5-8]. The Zn3d core level photoemission peak is sharp and its full width at half maximum (FWHM) is exactly the same as for a single $\mathrm{ZnO}$ crystal and equals $1.6 \mathrm{eV}$. The sharpness of the peak indicates that manganese does not lead to the disorder in the $\mathrm{ZnO}$ cation sublattice.

The scanning Auger microprobe measurements were taken for $\mathrm{Mn} / \mathrm{ZnO}$ system after annealing. Auger spectra from the surface were measured during ion etching of a small spot of the crystal. There was no Mn capping layer and $\mathrm{Zn}$ was abundant at the surface. The $\mathrm{Mn} / \mathrm{Zn}$ ratio measured on the surface was 0.43 . During etching the oxygen concentration was constant and $\mathrm{Zn}$ gradually replaced Mn. The transition region width had an extension of about $20 \AA$ with gradually lower Mn concentration.

In Figs. 2a, 3a, and 4a we present a set of EDCs taken for photon energies 46-60 eV (across the resonance) after deposition of $2 \mathrm{Mn}$ ML (Fig. 2), $4 \mathrm{Mn}$ ML (Fig. 3), and after annealing of $\mathrm{Mn} / \mathrm{ZnO}(000 \underline{1})$ system in $500^{\circ} \mathrm{C}$ by 18 hours (Fig. 4). In Figs. 2b-4b one can follow redistribution of the photoemission intensity caused by manganese deposition and annealing.

a) $\mathrm{ZnO}(0001)+2 \mathrm{ML} \mathrm{Mn}$

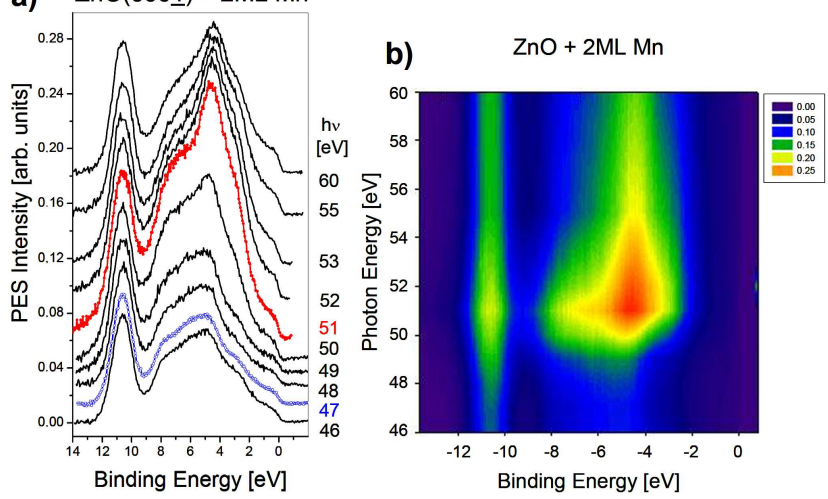

Fig. 2. (a) EDCs for $\mathrm{Mn} / \mathrm{ZnO}(000 \underline{1})$ system measured after deposition of $2 \mathrm{Mn}$ ML. Spectra normalized to the maximum. (b) The contour graph made from EDCs taken after deposition of $2 \mathrm{Mn}$ ML and normalized to the photon flux. 

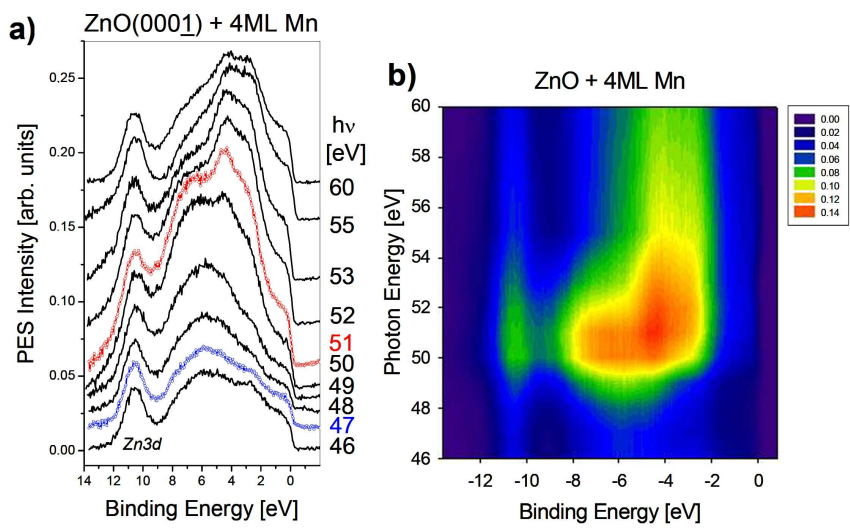

Fig. 3. (a) EDCs for $\mathrm{Mn} / \mathrm{ZnO}(000 \underline{1})$ system measured after deposition of $4 \mathrm{Mn}$ ML. Spectra normalized to the maximum. (b) The contour graph made from EDCs taken after deposition of $4 \mathrm{Mn}$ ML and normalized to the photon flux.

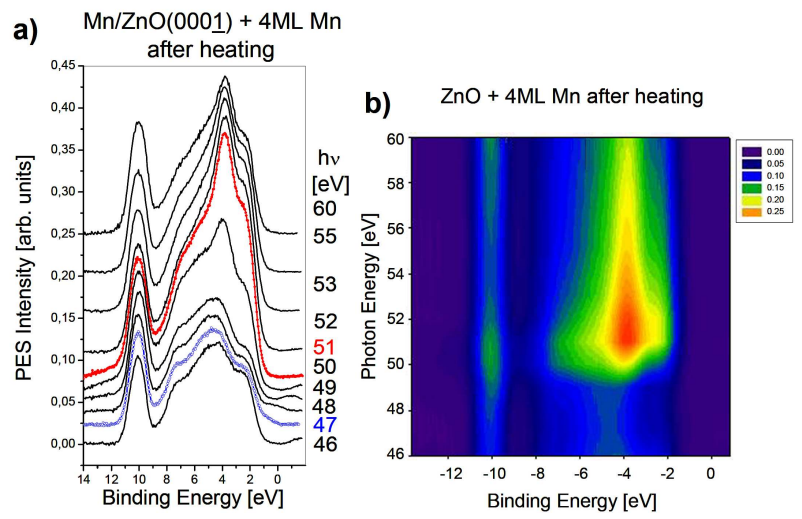

Fig. 4. (a) EDCs for $\mathrm{Mn} / \mathrm{ZnO}(000 \underline{1})$ system measured after deposition of $4 \mathrm{Mn} \mathrm{ML}$ and annealing the system to $500^{\circ} \mathrm{C}$. Spectra normalized to the maximum. (b) The contour graph made from EDCs taken after annealing to $500^{\circ} \mathrm{C}$. Spectra normalized to the photon flux.

The experimental Mn3d partial density of states is presented in Fig. 5. The $\Delta \mathrm{EDC}=\mathrm{EDC}(51 \mathrm{eV})-\mathrm{EDC}(47 \mathrm{eV})$ spectra consists of three structures typically found in II-VI ternary compounds with manganese [5-8]. The main structure is situated at 3.8-4.5 eV, valence structure at $0-3 \mathrm{eV}$ and a broad satellite between 5.5 and $9 \mathrm{eV}$ below the Fermi edge. For $\Theta=2$ and $4 \mathrm{ML}$ the low energy shoulder of the main peak contains two contributions: a metallic shoulder located at the Fermi edge and structure around $3 \mathrm{eV}$ below.

Electronic structure calculations are available for $\mathrm{Cd}_{1-x} \mathrm{Mn}_{x} \mathrm{Te}[9,10]$ ternary compound. They are also used to interpret the Mn-derived structures 

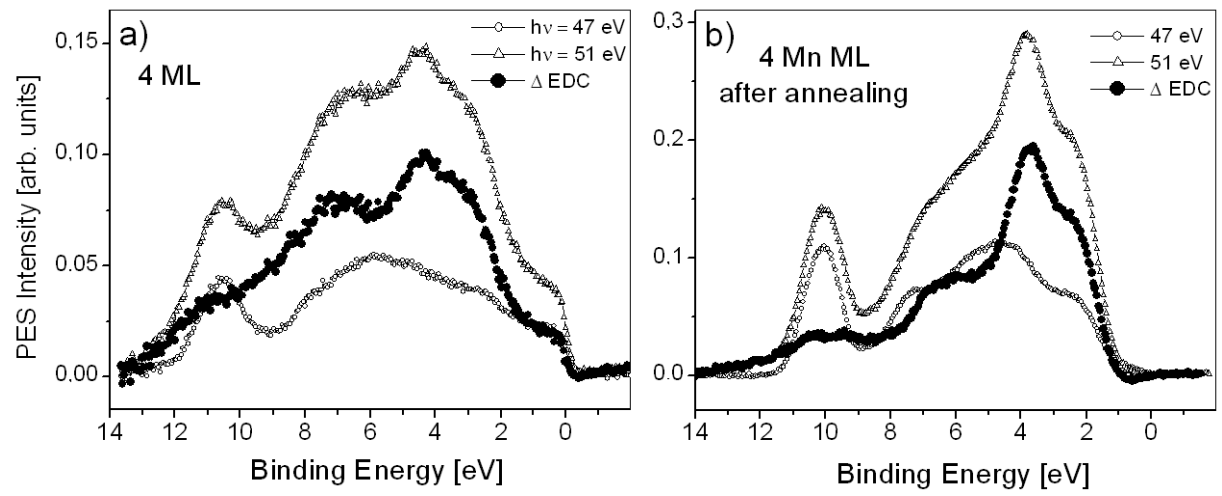

Fig. 5. EDCs and $\triangle E D C$ spectra (EDC at resonance minus EDC at anti-resonance) taken after deposition of $4 \mathrm{Mn}$ ML (a) and after annealing (b). Open triangles show resonant spectra $(h \nu=51 \mathrm{eV})$, open circles - anti-resonance spectra $(h \nu=47 \mathrm{eV})$, and solid circles - calculated EDC spectra.

of other Mn-substituted II-VI compounds. The main peak in the overall structure of the Mn3d partial density of states represents a part of the Mn3d electrons only weakly influenced by hybridization. The valence structure presumably originates from the Mn3d states hybridized with the valence band. The satellite structure is not related to the partial density of Mn3d states, but represents a shake-up-like satellite of the main Mn3d peak, which is not described in the one-electron picture.

The binding energy of the main $\mathrm{Mn} 3 d$ structure of $\mathrm{Mn} / \mathrm{ZnO}$ system is $4.4 \mathrm{eV}$ and $4.38 \mathrm{eV}$ for $\Theta=2$ and $4 \mathrm{ML}$, respectively, and $3.8 \mathrm{eV}$ below the Fermi edge after annealing. The intensity of satellite/main peak ratio changes from 0.33 for $\Theta=0.4 \mathrm{ML}$ to 0.89 for $\Theta=4 \mathrm{ML}$ and drops to 0.43 after annealing. The relative satellite/main intensity of the Mn3d peak in $\mathrm{ZnMnY}(\mathrm{Y}=\mathrm{S}, \mathrm{Se}, \mathrm{Te})$ compounds was regarded in dependence of $V_{p d}$ hybridization parameter [5]. The 0.43 value obtained for $\mathrm{ZnMnO}$ alloy follow the trend toward higher hybridization as we move up in the Periodic Table, which was found in ZnY compounds (Table).

TABLE

The energy of the main Mn3d peak and branching ratio of the satellite to the main structure in $\mathrm{Zn}-\mathrm{VI}$ ternary alloys with $\mathrm{Mn}$. The data of $\mathrm{ZnY}(\mathrm{Y}=\mathrm{Te}, \mathrm{Se}, \mathrm{S})$ are taken from Ref. [5].

\begin{tabular}{c|c|c}
\hline \hline Sample & $E_{\text {main }}[\mathrm{eV}]$ & Satellite/Main ratio \\
\hline $\mathrm{Zn}_{0.68} \mathrm{Mn}_{0.32} \mathrm{Te}$ & $3.8^{*}$ & 1.05 \\
$\mathrm{Zn}_{0.81} \mathrm{Mn}_{0.19} \mathrm{Se}$ & $3.7^{*}$ & 0.90 \\
$\mathrm{Zn}_{0.81} \mathrm{Mn}_{0.19} \mathrm{~S}$ & $3.5^{*}$ & 0.4 \\
$\mathrm{Mn} / \mathrm{ZnO}$ after annealing & $3.0^{*}$ & 0.43
\end{tabular}

${ }^{*}$ The binding energy is given in respect to the valence band edge. 
According to the results obtained for $\mathrm{ZnMnY}(\mathrm{Y}=\mathrm{S}, \mathrm{Se}, \mathrm{Te})$ ternary compounds $V_{p d}$, a parameter, describing the amount of the Mn3d-anion $p$ hybridization, increases with a decrease in satellite/main branching ratio. Therefore for $\mathrm{Mn} / \mathrm{ZnO}$ system we may expect a hybridization effect comparable to ZnMnS compound and higher than in the case of ZnMnSe or ZnMnTe. It is also higher than the hybridization effect in the widely investigated $\mathrm{Ga}_{1-x} \mathrm{Mn}_{x}$ As compounds [11].

It is worth mentioning that the experimental Mn3d partial density of states presented here very well follows the trend found in the chalogenide-based semiconductors [5]. The similarity is much better than in the case of $\mathrm{Zn}_{1-x} \mathrm{Mn}_{x} \mathrm{O}$ thin films obtained by pulsed-laser deposition [12].

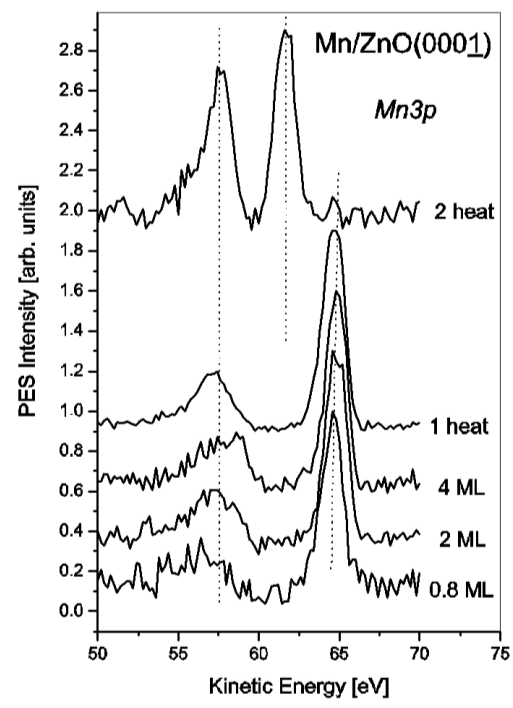

Fig. 6. The Mn $3 p$ electron state measured with photon energy of $110 \mathrm{eV}$. Spectra show two Mn3 $p$ components chemically shifted by $4 \mathrm{eV}$.

In Fig. 6 we show the photoemission spectra of Mn3p core level measured at $h \nu=110 \mathrm{eV}$ after deposition of $0.8 \mathrm{ML}, 2 \mathrm{ML}$, and $4 \mathrm{Mn} \mathrm{ML}$, and after annealing. EDCs show two Mn3p features observed at kinetic energies $57.5 \mathrm{eV}$ and $64.9 \mathrm{eV}$ before annealing and $57.7 \mathrm{eV}$ and $61.7 \mathrm{eV}$ after this process. The intensity of the structure observed at lower kinetic energy increases after annealing and presumably it consists of two photoemission peaks. This is the evidence that we have at least two manganese states in the $\mathrm{Mn}-\mathrm{ZnO}$ interface region.

\section{Summary}

In conclusion, we have measured $\mathrm{Mn} / \mathrm{ZnO}(0001)$ system by synchrotron radiation photoemission. After deposition of $4 \mathrm{ML}$ of manganese on clean $\mathrm{ZnO}(000 \underline{1})$ 
surface and annealing up to $500^{\circ} \mathrm{C}$ the metallic shoulder at the Fermi edge disappeared and no Mn capping layer has been found at the surface. The intensity of satellite/main peak in the experimental Mn3d partial density of states was determined as 0.43. This is evidence that the hybridization effect in $\mathrm{Zn}_{1-x} \mathrm{Mn}_{x} \mathrm{O}$ surface alloy is comparable to $\mathrm{Zn}_{1-x} \mathrm{Mn}_{x} \mathrm{~S}$ and much higher than in $\mathrm{Zn}_{1-x} \mathrm{Mn}_{x} \mathrm{Se}$, $\mathrm{Zn}_{1-x} \mathrm{Mn}_{x}$ Te, and $\mathrm{Ga}_{1-x} \mathrm{Mn}_{x}$ As semimagnetic compounds. The Mn3p spectra of annealed $\mathrm{Mn} / \mathrm{ZnO}$ system show two contributions, which is evidence of two manganese states in ternary alloy in the $\mathrm{ZnMnO}$ subsurface region.

\section{Acknowledgments}

The work was supported by the State Committee for Scientific Research project PBZ-KBN-044/P03/2001 (Poland). Measurements at MAX-Lab Swedish national synchrotron radiation facility have been supported by the European Community within the "Access to Research Infrastructure Action of the Improving Human Potential Programme".

\section{References}

[1] N. Ohashi, Y. Terada, T. Ohgaki, S. Tanaka, T. Tsurumi, O. Fukunaga, H. Haneda, J. Tanaka, Jpn. J. Appl. Phys. 38, 5028 (1999).

[2] Z. Jin, T. Fukumura, M. Kawasaki, K. Ando, H. Saito, T. Sekiguchi, Y.Z. Yoo, M. Murakami, Y. Matsumoto, T. Hagesawa, H. Koinuma, Appl. Phys. Lett. 78, 3824 (2001).

[3] T. Fukumura, Z. Jin, A. Ohtomo, H. Koinuma, M. Kawasaki, Appl. Phys. Lett. 75, 3366 (1999).

[4] J. Sadowski, J.Z. Domagala, J. Bak-Misiuk, S. Kolesnik, M. Sawicki, K. Swiatek, J. Kanski, L. Ilver, V. Ström, J. Vac. Sci.Technol. B 18, 1697 (2000).

[5] R. Weidemann, H.-E. Gumlich, M. Kupsch, H.-U. Middelmann, U. Becker, Phys. Rev. B 45, 1172 (1992)

[6] M. Taniguchi, K. Mimura, H. Sato, J. Harada, K. Miyazaki, H. Namatame, Phys. Rev. B 51, 6932 (1995).

[7] A. Wall, A. Raisanen, G. Haugstad, L. Vanzetti, A. Franciosi, Phys. Rev. B 44, 8185 (1991).

[8] N. Happo, H. Sato, K. Mimura, S. Hosokawa, M. Taniguchi, Y. Ueda, M. Koyama, Phys. Rev. B 50, 12211 (1994).

[9] S.-H. Wei, A. Zunger, Phys. Rev. B 35, 2340 (1987).

[10] O. Gunnarsson, O.K. Andersen, O. Jepsen, J. Zaanen, Phys. Rev. B 39, 1708 (1989).

[11] J. Okabayashi, A. Kimura, T. Mizokawa, A. Fujimori, T. Hayashi, M. Tanaka, Phys. Rev. B 59, R2486 (1999).

[12] T. Mizokawa, T. Nambu, A. Fujimori, T. Fukumura, M. Kawasaki, Phys. Rev. B 65, 85209 (2002). 